\title{
Erratum to: SPE and LC-MS/MS determination of 14 illicit drugs in surface waters from the Natural Park of L'Albufera (València, Spain)
}

\author{
Pablo Vazquez-Roig ${ }^{1}$. Vicente Andreu ${ }^{2}$. Cristina Blasco ${ }^{1}$ Yolanda Picó ${ }^{1}$
}

Received: 5 May 2015 / Accepted: 5 May 2015 /Published online: 3 July 2015

(C) Springer-Verlag Berlin Heidelberg 2015

Erratum to: Anal Bioanal Chem

DOI: $10.1007 / \mathbf{s 0 0 2 1 6 - 0 1 0 - 3 7 2 0 - x}$

We should like to call your attention to the fact that the Electronic Supplementary Material linked to this article was incorrect. It corresponds to the supplementary content of the article [1].

\section{References}

1. Malik AK, Blasco C, Picó Y (2010) Liquid chromatography-mass spectrometry in food safety, J Chromatogr A 2010 1217:4018-4040. doi:10.1016/j.chroma.2010. 03.015

Please find below the correct data:

Electronic Supplementary Material

The online version of the original article can be found at http://dx.doi.org/ 10.1007/s00216-010-3720-x.

Yolanda Picó

yolanda.pico@uv.es

1 Laboratori de Nutrició i Bromatologia, Facultat de Farmàcia,

Universitat de València, Av, Vicent Andrés s/n,

46100 Burjassot, València, Spain

2 Centro de Investigaciones sobre Desertificacion-CIDE

(CSIC-UV-GV), Camí de la Marjal s/n, 46470 Albal, València, Spain 


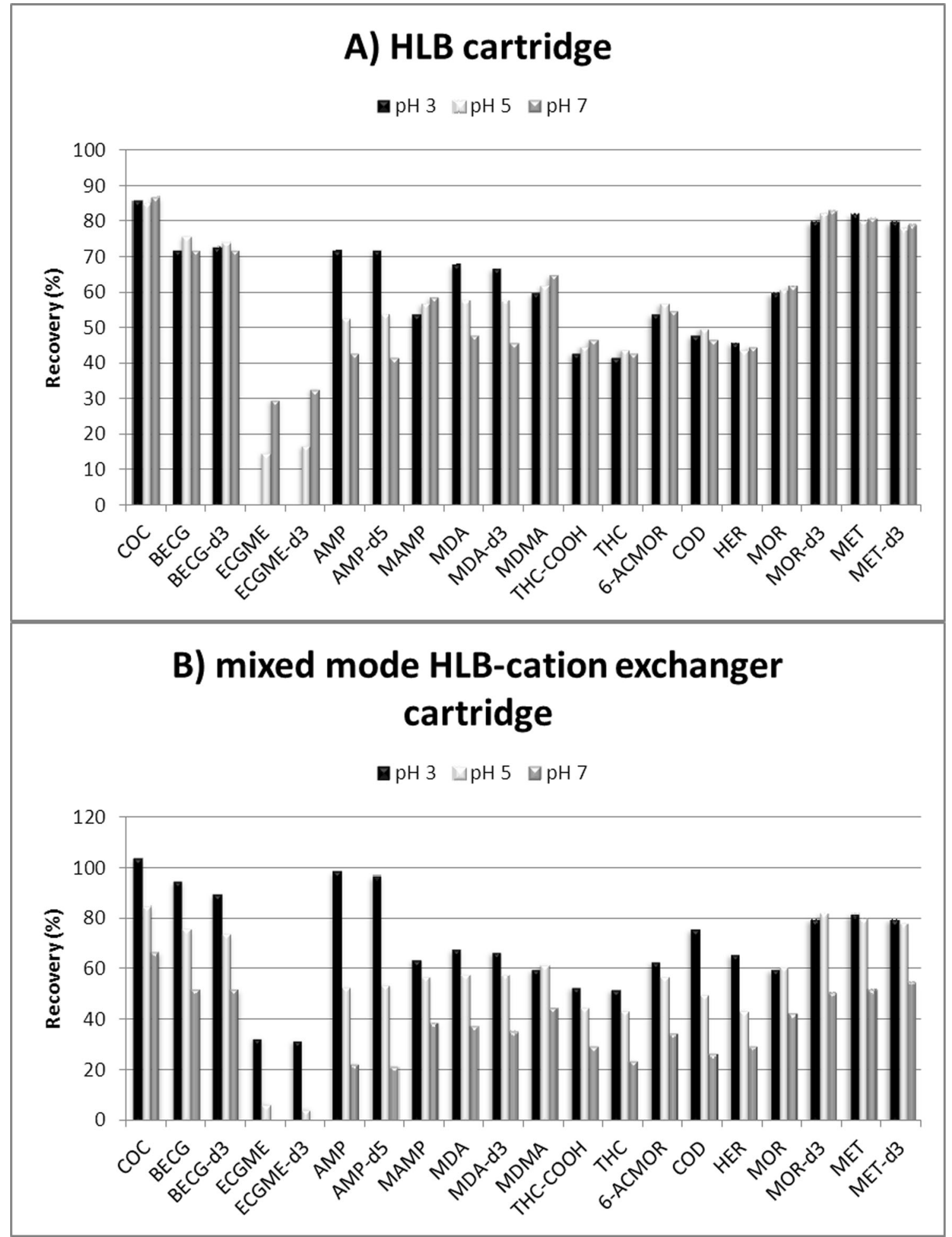

Fig. S1 Influence of $\mathrm{pH}$ adjustment, (pH 3, $\mathrm{pH} 5$ and $\mathrm{pH} 7$ ), on the absolute recoveries using (A) SupelSelect HLB SPE cartridges and (B) Strata-XC (matrix surface water from L'Albufera and spiking level $50 \mathrm{ng} / \mathrm{L}$ ). 


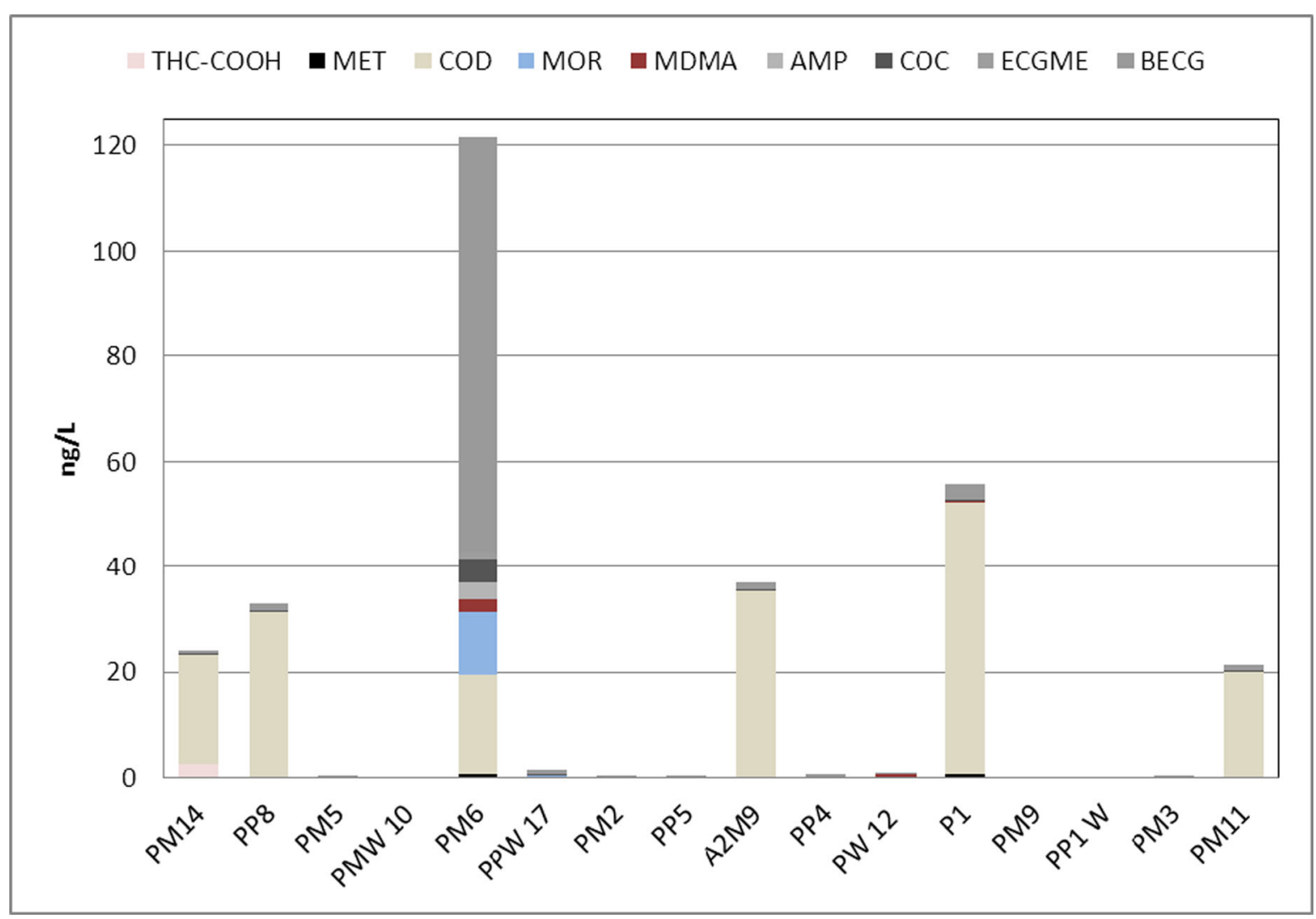

Fig. S2 Cummulative levels ng/L of drugs of abuse in the surface waters of the Natural Park of L'Albufera.

Table S1 Absolute recoveries (in \%) for Oasis HLB, Strata X and Strata-XCW cartridges with different sorbent mass and sample volume. (matrix: L'Albufera superficial and spiking level $50 \mathrm{ng} / \mathrm{L}$ )

\begin{tabular}{|c|c|c|c|c|c|c|c|c|c|c|c|c|}
\hline \multirow[t]{3}{*}{ Compound } & \multicolumn{4}{|c|}{ Oasis HLB (pH 7) } & \multicolumn{4}{|c|}{ Strata-X (pH 7) } & \multicolumn{4}{|c|}{ Strata-XCW (pH 2) } \\
\hline & \multicolumn{2}{|c|}{$60 \mathrm{mg} / 3 \mathrm{~mL}$} & \multicolumn{2}{|c|}{$200 \mathrm{mg} / 6 \mathrm{~mL}$} & \multicolumn{2}{|c|}{$60 \mathrm{mg} / 3 \mathrm{~mL}$} & \multicolumn{2}{|c|}{$200 \mathrm{mg} / 6 \mathrm{~mL}$} & \multicolumn{2}{|c|}{$60 \mathrm{mg} / 3 \mathrm{~mL}$} & \multicolumn{2}{|c|}{$200 \mathrm{mg} / 6 \mathrm{~mL}$} \\
\hline & $50 \mathrm{~mL}$ & $250 \mathrm{~mL}$ & $50 \mathrm{~mL}$ & $250 \mathrm{~mL}$ & $50 \mathrm{~mL}$ & $250 \mathrm{~mL}$ & $50 \mathrm{~mL}$ & $250 \mathrm{~mL}$ & $50 \mathrm{~mL}$ & $250 \mathrm{~mL}$ & $50 \mathrm{~mL}$ & $250 \mathrm{~mL}$ \\
\hline 6ACMOR & 54 & 50 & 65 & 63 & 57 & 45 & 62 & 57 & 42 & 34 & 51 & 48 \\
\hline AMP & 43 & 35 & 55 & 50 & 43 & 31 & 53 & 43 & 43 & 32 & 54 & 49 \\
\hline AMP-d ${ }_{5}$ & 42 & 38 & 50 & 51 & 42 & 30 & 50 & 42 & 42 & 35 & 49 & 44 \\
\hline BECG & 72 & 66 & 84 & 79 & 76 & 64 & 84 & 76 & 62 & 53 & 71 & 69 \\
\hline BECG-d ${ }_{3}$ & 74 & 67 & 85 & 80 & 74 & 62 & 86 & 74 & 54 & 36 & 65 & 68 \\
\hline $\mathrm{COC}$ & 86 & 81 & 98 & 92 & 85 & 73 & 95 & 85 & 40 & 28 & 51 & 49 \\
\hline COD & 48 & 43 & 60 & 56 & 50 & 38 & 56 & 50 & 40 & 32 & 49 & 47 \\
\hline ECGME & 30 & 26 & 39 & 32 & 30 & 18 & 32 & 30 & 24 & 18 & 32 & 33 \\
\hline ECGME-d $_{3}$ & 33 & 27 & 42 & 37 & 33 & 21 & 34 & 33 & 23 & 12 & 34 & 35 \\
\hline HER & 46 & 39 & 56 & 53 & 44 & 32 & 50 & 44 & 40 & 34 & 48 & 46 \\
\hline MAMP & 54 & 44 & 63 & 59 & 54 & 42 & 58 & 54 & 54 & 45 & 62 & 58 \\
\hline MDA & 48 & 41 & 53 & 48 & 48 & 36 & 55 & 48 & 48 & 40 & 56 & 53 \\
\hline MDMA & 46 & 39 & 52 & 47 & 46 & 34 & 56 & 46 & 46 & 38 & 54 & 52 \\
\hline MET & 60 & 54 & 70 & 60 & 60 & 48 & 70 & 62 & 60 & 53 & 68 & 69 \\
\hline MET-d $_{3}$ & 82 & 78 & 93 & 90 & 80 & 68 & 83 & 80 & 70 & 64 & 79 & 76 \\
\hline MOR & 80 & 78 & 90 & 88 & 78 & 66 & 80 & 78 & 70 & 62 & 82 & 74 \\
\hline MOR-d $\mathrm{d}_{3}$ & 60 & 51 & 72 & 70 & 61 & 49 & 66 & 61 & 55 & 46 & 65 & 60 \\
\hline THC & 80 & 76 & 94 & 89 & 82 & 70 & 85 & 82 & 60 & 57 & 73 & 65 \\
\hline THC-COOH & 42 & 36 & 49 & 48 & 44 & 32 & 53 & 44 & 23 & 14 & 34 & 36 \\
\hline
\end{tabular}

